\title{
Transplantation of Autologous Bone Marrow-Derived Stromal Cells into Injured Spinal Cord Enhances Glucose Metabolism and Promotes Functional Recovery
}

\author{
Jesús Vaquero ${ }^{1 *}$, Mercedes Zurita ${ }^{1}$, Jorge Mucientes ${ }^{2}$, Gregorio Rodríguez-Boto ${ }^{1}$ \\ and Cecilia Fernández-Mateos ${ }^{1}$
}

${ }^{1}$ Service of Neurosurgery, Puerta de Hierro-Majadahonda Hospital, Autonomous University, Spain

${ }^{2}$ Service of Nuclear Medicine, Puerta de Hierro-Majadahonda Hospital, Autonomous University, Spain

*Corresponding author: Jesús Vaquero, Service of Neurosurgery, Puerta de Hierro-Majadahonda Hospital, Autonomous University, Manuel de Falla 1, 28222-Madrid, Spain

\begin{abstract}
The authors report the case of a 40 -year-old man who suffered paraplegia from 2013, due to shot by firearm. In april 2017 he underwent cell therapy consisting of administration of autologous bone marrow stromal cells (BMSCs) into injured spinal cord and subarachnoid space by lumbar puncture. Throughout one year of follow-up, the patient experienced clear improvement in sensitivity, motor, and sphincter functions. After BMSCs administration, studies with $18 \mathrm{~F}$-fluorodeoxyglucose positron emission tomography (18F-FDG-PET) suggested an enhancement in glucose uptake at level of injured spinal cord, at least in the course or the first 6 months after the start of cell therapy. This case supports previous observations about the improvements obtained by cell therapy with autologous BMSCs in chronic paraplegic patients, and suggests that a possible increase in tissue metabolism, mediated by the presence of BMSCs into injured spinal cord, can be one of the causes of early clinical improvements.
\end{abstract}

\section{Keywords}

Brain glucose metabolism, Cell therapy, Spinal cord injury

In recent years, cell therapy, mainly using autologous bone marrow stromal cells (BMSCs), is configured as a hope for patients suffering neurological sequelae due to spinal cord injury (SCI) $[1,2]$. Although a paracrine effect, by releasing neurotrophic factors by transplanted stem cells is considered as the main cause of improvement, it is difficult to answer the multiple questions posed by the efficacy of these techniques.
Recently, we have described that after intrathecal administration of BMSCs a great and progressive increase in cerebral glucose metabolism occur, a finding that could be an explanation for cognitive improvement in patients suffering severe traumatic brain injury (TBI) [3].

We describe here a paraplegic patient showing progressive improvement after surgical administration of autologous BMSCs into injured spinal cord and subarachnoid space. A study with $18 \mathrm{~F}$-fluorodeoxyglucose positron emission tomography (18F-FDG-PET) suggested the presence of glucose uptake enhancement in the zone of SCl.

\section{Case Report}

\section{History and examination}

Our patient suffered a SCl on November, 12, 2013, by a shot not accompanied by vertebral fracture. The patient was not operated and since the lesion he remained without being able to make any kind of voluntary movement in both legs, with areas of reduced sensitivity, at light touch exploration below the Th11 level. He was graded as a B patient in the impairment scale from the American Spinal Injury Association (ASIA) [4], with a Th 9 neurological level. This situation remained unchanged until 2017 . The patient then presented a score of 3 in the modified Ashworth scale

Citation: Vaquero J, Zurita M, Mucientes J, Rodríguez-Boto G, Fernández-Mateos C (2018) Transplantation of Autologous Bone Marrow-Derived Stromal Cells into Injured Spinal Cord Enhances Glucose Metabolism and Promotes Functional Recovery. Int J Stem Cell Res Ther 5:057. doi. org/10.23937/2469-570X/1410057

Accepted: December 22, 2018: Published: December 24, 2018

Copyright: (C) 2018 Vaquero J, et al. This is an open-access article distributed under the terms of the Creative Commons Attribution License, which permits unrestricted use, distribution, and reproduction in any medium, provided the original author and source are credited. 

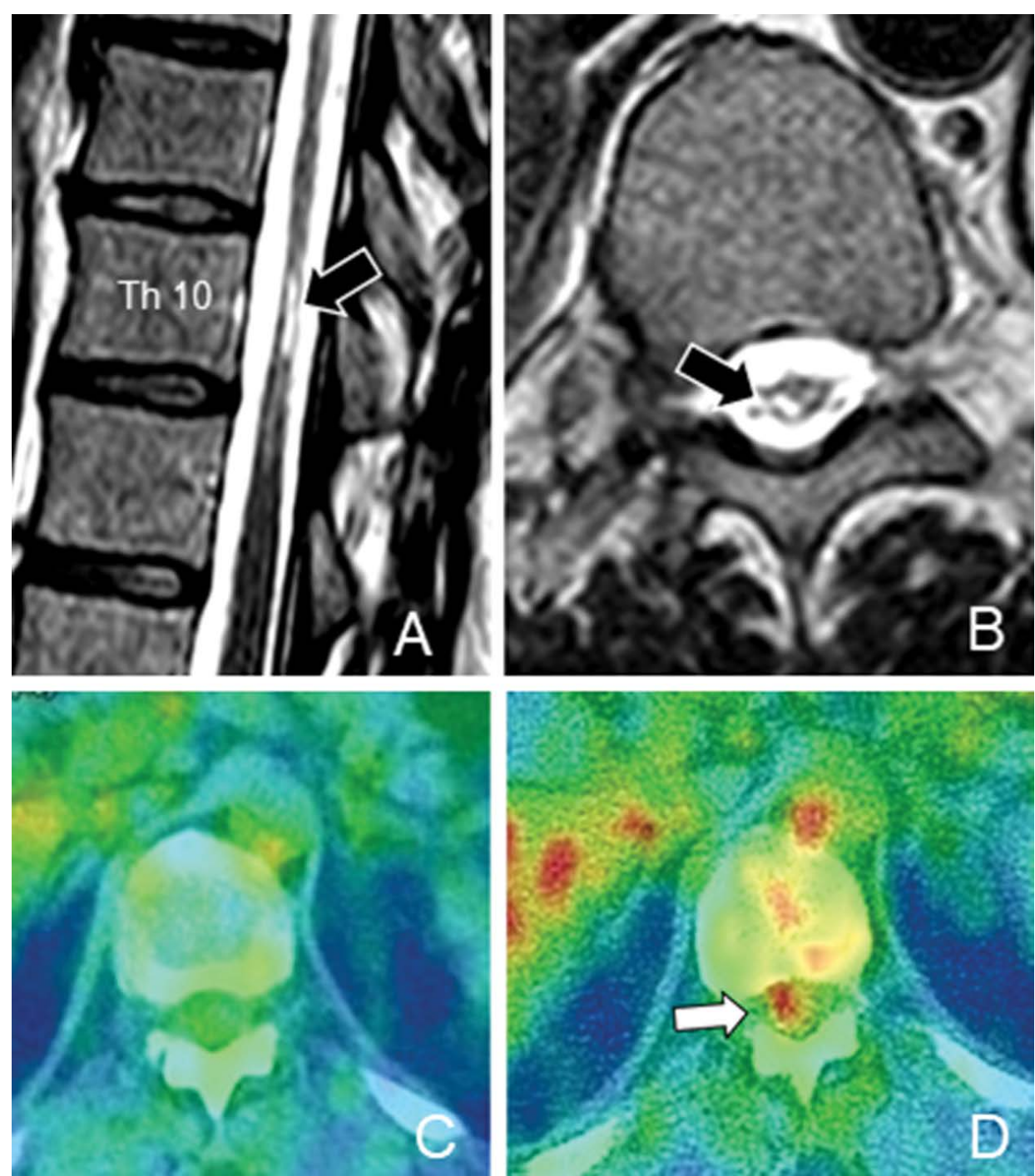

Figure 1: $A, B)$ MRI (T2-weighted images) showing gliosis in injured spinal cord (arrows); C) Axial view in the 18F-FDG-PET study, previously to cell therapy, showing absence of hypermetabolism into spinal canal at level of SCl; D) One week after BMSCs administration, hypermetabolism into injured spinal cord can be seen (arrow).

[5] and bladder sphincter control had a score of 2 in the Geffner scale [6]. Magnetic Resonance Image (MRI) showed a zone of spinal cord atrophy in the lower part of the thoracic spinal cord, with hyperintensity on T2 weighted images that suggested gliosis, mainly at level of Th10 (Figure 1A and Figure 1B). 18F-FDG-PET showed absence of isotopic captation inside the spinal canal. (Figure $1 \mathrm{C}$ and Figure 2B).

\section{Treatment}

At this time, a treatment of cell therapy with NC1 medicament was performed, after authorization of the Spanish Agency for Medicaments and Health Products (AEMPS), and Medical Management of Puerta de Hierro-Majadahonda Hospital. NC1 medicament, was developed in the clean room of neurosurgical service, and currently approved as a medicament under clinical investigation (PEI No. 12-141). It consists of expanded autologous BMSCs, with autologous plasma as its excipient. Techniques for culture and expansion of BMSCs, phenotypic characterization, formulation and packaging have been previously described $[1,2]$.
After a laminectomy and durotomy, the zone of injured spinal cord was exposed and approximately 300 million of autologous BMSCs were administered in previously selected points and adjacent subarachnoid space with the aid of a microinjection pump (mod 310, Stoeling Co, Wood Dale, Illinois, USA) connected to a $100 \mathrm{~mL}$ Hamilton syringe, with a 20-gauge needle and a microinjection rate of $10 \mathrm{~mL} / \mathrm{min}$ (Figure $2 \mathrm{~A}$ ). The microinjection pump was attached to a device previously described [7]. Three months later a lumbar puncture was performed, and an additional dose of 100 million BMSCs were administered into subarachnoid space.

\section{Postoperative course}

During the surgery, or in the postoperative course, no adverse events were observed. A PET study with 18F-FDG was performed one week after surgery, showing a focus of metabolic hyperactivity at the level Th10, where the MSCs were administered (Figures 1D and Figure $2 \mathrm{C}$ ), and increase of metabolic activity in the whole brain cortex. Two months later, a new PET showed two 

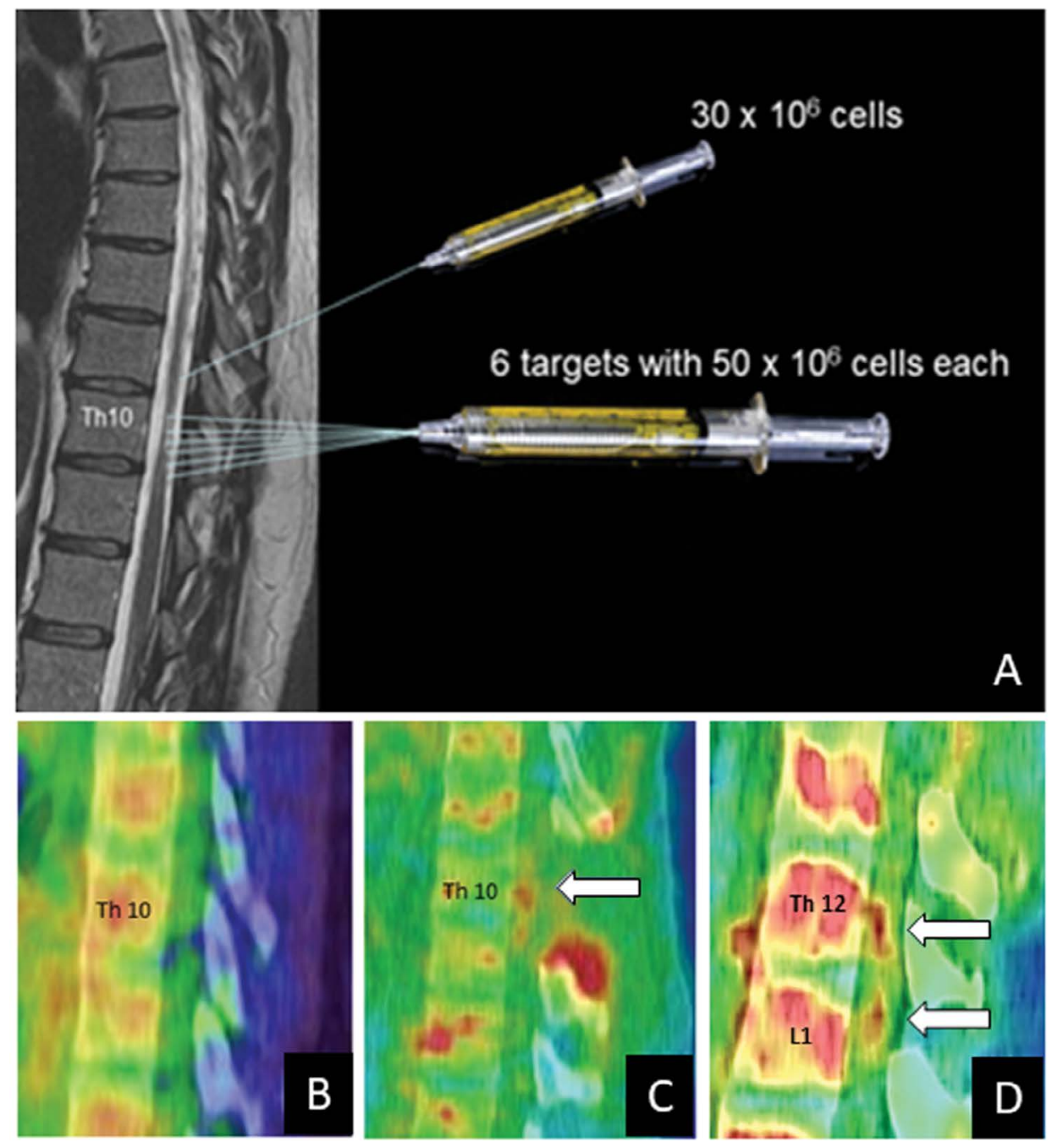

Figure 2: A) Surgical planning for the administration of our medicament into damaged spinal cord and subarachnoid space; B) Sagittal image in the 18F-FDG-PET study performed previously to cell therapy, showing absence of hypermetabolism into spinal canal; C) One week after laminectomy (arrow) and BMSCs administration, hypermetabolism can be seen into injured spinal cord at level of cell administration; D) Two months later, two new foci of hypermetabolism can be seen at Th12 and L1 levels.

new foci of metabolic activity at Th12 and L1 vertebral level (Figure 2D), and persisted the enhancement in brain metabolism. One year after the end of cell therapy, the pattern of glucose uptake was considered normal in the brain, without foci of hypermetabolism into spinal canal.

Voluntary movements in the toes of the feet were initiated two weeks after surgery, and in the course of the following months, the patient showed clear and progressive motor improvement, being able to stand up with the help of canes one year after surgery, and pedal voluntarily on a stationary bike, but without being able to take steps. Supplementary Movie show the motor improvement obtained during the followup. The patient described also progressive increase in infralesional sensitivity and in bladder control, with a score of 5 in the Geffner scale [6] one year after surgery.

\section{Discussion}

In recent years evidence has been obtained that the transplantation of autologous BMSCs can be useful to improve the quality of life of patients suffering $\mathrm{SCl}$ $[1,2,8-11]$. The mechanisms by which this improvement occurs are not clear, but it is accepted that an important role is the release of trophic substances from the BMSCs that act by a paracrine mechanism and activate nervous circuits, anatomically present after $\mathrm{SCl}$, but not functioning. Recently, these cell therapy techniques have been applied to patients with TBI and there are already some observations that suggest a beneficial effect, possibly through an activation of cerebral metabolism for glucose uptake, measured by 18F-FDG-PET [3]. These observations support previous studies in rodents showing that stem cell transplantation after cerebral infarction [12] or TBI [13] achieves an enhancement of local glucose metabolism, being related this finding to a neurological improvement.

At present, we consider that enhancement of brain glucose uptake is an effect of the presence of BMSCs into subarachnoid space and its subsequent diffusion to the whole brain by cerebrospinal fluid (CSF) [3]. In our present case report we have studied the 
possibility that neurological improvements obtained after intrathecal administration of BMSCs in paraplegic patients can be related to an enhancement in metabolic reactivation in injured spinal cord. Although, at present, technical limitations prevent an accurate localization of hypermetabolism into spinal canal after 18F-FDG-PET studies, our findings suggest this possibility.

In conclusion, for the first time in humans, we present images suggesting metabolic enhancement into injured spinal cord after local transplantation of autologous BMSCs. This finding requires further studies and should be taken into account when interpreting neurological improvements in paraplegic patients subjected to this type of cell therapy.

\section{Disclosure}

The author report no conflict of interest concerning the material or methods used in this study or the findings specified in this paper.

\section{Acknowledgment}

We thank the institutions supporting our neurological cell therapy program, mainly Mapfre and Rafael del Pino Foundations.

\section{References}

1. Vaquero J, Zurita M, Rico MA, Bonilla C, Aguayo C, et al. (2016) An approach to personalized cell therapy in chronic complete paraplegia: The Puerta de Hierro phase I/II clinical trial. Cytotherapy 18: 1025-1036.

2. Vaquero J, Zurita M, Rico MA, Bonilla C, Aguayo C, et al. (2017) Repeated subarachnoid administrations of autologous mesenchymal stromal cells supported in autologous plasma improve quality of life in patients suffering incomplete spinal cord injury. Cytotherapy 19: 349-359.

3. Vaquero J, Zurita $M$, Bonilla $C$, Fernández C, Rubio JJ, et al. (2017) Progressive increase in brain glucose metabolism after intrathecal administration of autologous mesenchymal stromal cells in patients with diffuse axonal injury. Cytotherapy 19: 88-94.
4. Kirshblum SC, Burns SP, Biering-Sorensen F, William Donovan, Daniel E Graves, et al. (2011) International standards for neurological classification of spinal cord injury (revised 2011). J Spinal Cord Med 34: 535-546.

5. Bohannon RW, Smith MB (1987) Interrater reliability of a modified Ashworth scale of muscle spasticity. Phys Ter 67: 206-207.

6. Geffner LF, Santacruz P, Izurieta M, Flor L, Maldonado B, et al. (2008) Administration of autologous bone marrow stem cells into spinal cord injury patients via multiple routes is safe and improves their quality of life: Comprehensive case studies. Cell Transplant 17: 1277-1293.

7. Vaquero J, Zurita M, Fernández C, Cotua C (2016) An inexpensive attachment device for cell therapy administration into injured spinal cord. World Neurosurg 96: 440-443.

8. Vaquero J, Zurita M, Rico MA, Aguayo C, Bonilla C, et al. (2018) Intrathecal administration of autologous mesenchymal stromal cells for spinal cord injury: Safety and efficacy of the 100/3 guideline. Cytotherapy 20: 806-819.

9. Vaquero J, Zurita M, Rico MA, Aguayo C, Fernandez C, et al. (2018) Cell therapy with autologous mesenchymal stromal cells in posttraumatic syringomyelia. Cytotherapy 20: 796-805.

10. Tapiador N, Sevilla M, Zurita M, Fernández-Mateos $\mathrm{C}$, Vaquero J. Intrathecal administration of autologous mesenchymal stromal cells for incomplete spinal cord injury: Long-term outcome according to the ASIA assessment. Spinal Disease and Research.

11. Guadalajara Labajo H, León Arellano M, Vaquero Crespo J, Valverde Núñez I, García-Olmo D (2018) Objective demonstration of improvement of neurogenic bowel dysfunction in a case of spinal cord injury following stem cell therapy. J Surg Case Reports 11: 300.

12. Miyamoto M, Kuroda S, Zhao S, Magota K, Shichinohe $\mathrm{H}$, et al. (2013) Bone marrow stromal cell transplantation enhances recovery of local glucose metabolism after cerebral infarction in rats; a serial 18F-FDG PET study. J Nucl Med 54: 145-150.

13. Park BN, Yoon JK, An YS (2013) Bone marrow mesenchymal stem cell transplantation in acute brain trauma. Improvement of brain glucose metabolism in a rat model. Nuklearmedizin 52: 192-197. 